\title{
Numerical Investigation on the effect of Blood pressure on Wall Shear Stress and Vorticity
}

\author{
NITESH KUMAR \\ Department of Mechanical Engineering., Manipal Institute of Technology, Manipal, Manipal Academy of \\ Higher Education, Manipal, INDIA
}

Abstract - Pulsatile blood flow through the human carotid artery is studied using Computational Fluid Dynamics (CFD) to investigate the effect of blood rheology on the hemodynamic parameters. The carotid artery model used is segmented and reconstructed from the Magnetic Resonance Images (MRI) of a specific patient. The results of a non-Newtonian (Carreau-Yasuda) model and a Newtonian model are studied and compared. The results are represented for each peak systole where it is observed that there is significant variation in the spatial parameters between the two models considered in the study. Comparison of local shear stress magnitude in different branches namely Common Carotid Artery (CCA), Internal Carotid Artery (ICA) and External Carotid Artery (ECA) show that the shear thinning property of blood influences the Wall Shear Stress (WSS) variation. This is observed in branches where there is reduction in diameter and where the diameter reduces due to plaque deposition and also in the region where there is flow recirculation like carotid sinus.

Keywords - Carotid artery, Newtonian and Carreau - Yasuda, Wall shear stress, Fluid Structure Interaction, Vortex flow.

Received: December 23, 2020. Revised: April 2, 2021. Accepted: April 15, 2021. Published: April 30, 2021.

\section{Introduction}

A THEROSCLEROSIS is the most common cardiovascular disease (CVD) causing increased morbidity in many industrialized countries [1]. Although atherosclerosis is a general disease involving several factors, it preferentially affects the wall of the vessel bifurcations [2]. The reason behind this focal nature is due to the local hemodynamic factors such as Wall shear stress and flow recirculation in those regions [3]. Atherosclerosis in large arteries is one of the major causes of stroke and is a cause for high risk of death and leading to major disabilities. It is mainly caused by ischemia or hemorrhage. Weakened cerebral hemodynamics is closely associated with carotid stenosis and blocking [4]. One of the symptoms is Transient Ischemic Attack (TIA). It was observed by Eliasziw et al [5] that concluded that risk of stroke was high for patients with TIA. Also it was nt related to degree of stenosis.. Stroke can also occur due to plaque rupture from a stenosed carotid artery, which is a perfect location for the development of atherosclerosis because of the arterial bifurcations leading to disturbed and complex flow [6]. The significant role played by hemodynamic constraints such as pressure, Wall Shear Stress (WSS) in the formation and progression of CVD makes it very essential to understand the hemodynamics in the carotid artery Blood is a non-Newtonian fluid. It comprises of various components such as platelets, white blood cells, red blood cell etc. [7], however researchers assumed blood as a Newtonian fluid [1] because in large vessels (lumen diameter varying from 1 to $3 \mathrm{~mm}$ ) the rate of shear are higher than $100 \mathrm{~s}-1[8]$. However, even in large arteries like carotid arteries, there are some regions where the diameters are significantly low resulting in low shear strain rate, where considering blood as a non-Newtonian fluid becomes necessary. Many researchers have studied different non-Newtonian blood viscosity models [9], out of which Carreau-Yasuda model was found suitable to simulate blood flow [1]. Jehangiri et al studied different non - Newtonian models to study the blood flow behavior and concluded that the power law is not suitable to capture the flow [12]. Lee et al also suggested that the rupture of plaque can be predicted depending upon the plaque cap thickness and other geometric properties [13].

Numerical investigation of haemodynamics in the carotid artery using Fluid structure interaction technique is an effective tool in understanding the flow dynamics and its effects on the arterial wall. The results from both structure and fluid are exchanged at the interface to obtain the desired results. In this study the behavior of flow of blood through stenosed carotid artery is subjected to normal High BP stage 1 , High BP stage 2, High BP stage 3and also hypotension. The purpose of this study was to enumerate the properties of different blood pressure ranges on the Wall Shear Stress (WSS) and vorticity and also in exploring the variations between Newtonian and non-Newtonian blood viscosity.

\section{Methodology}

\subsection{Image acquisition and reconstruction.}

3D model of patient specific carotid artery is constructed by selecting CT angio data followed by converting 2D CT scan images into 3D CAD model using image processing software like MIMICS 19 (Materialise, Leuven, Belgium). To avoid the stair-step artifact which generally appears on the curved surface of the model, scan interval is kept less than $2 \mathrm{~mm}$. The developed model had more than 300 slices each in coronal, sagittal, and axial planes. Using appropriate threshold ranging from -150 to $450 \mathrm{HU}$ in the present case, carotid artery is identified, and the segmentation process is carried out. Using automatic region growing the carotid bifurcation is segmented. As the FSI model consists of fluid and structural model representing blood and elastic artery, both are generated 
separately. The usual range of carotid intima media is in the range of 0.6 to $0.8 \mathrm{~mm}$. In this study the arterial wall thickness is assumed to be $0.7 \mathrm{~mm}$.

The FSI analysis was performed using Ansys CFX 19.0 and Ansys structural 19.0(ANSYS ${ }^{\circledR}$ Academic Research, Release 19.0). The artery was created as a linear elastic material with Elastic modulus of $0.9 \mathrm{MPa}$, and Poison's ratio of 0.45 . For the Newtonian viscosity model a density $1060 \mathrm{~kg} / \mathrm{m} 3$ and viscosity of $0.004 \mathrm{~Pa}-\mathrm{s}$ was used and the non - Newtonian consideration, Carreau - Yasuda model was chosen. The changes in WSS for different blood pressure ranges for both Newtonian and non - Newtonian models were considered and compared. Figures

\subsection{Modelling and computational setup.}

The general FSI problem involves the fluid and solid domains, interface conditions, and the relevant conditions for other boundaries. The fluid is considered laminar governed by the Navier - Stokes equations of incompressible flows in Arbitrary Lagrange Eulerian basis:

$\rho_{f}\left(\frac{\partial v_{f}}{\partial t}+v \cdot \nabla v\right)-\nabla \cdot \sigma_{f}=0, \nabla \cdot v=0$

in fluid domain. Where $\rho$ is persistent density and $\mathrm{v}$ is the velocity of the field. The flow is defined by pressure and velocity fields vf and $\mathrm{pf}$ respectively. The outside forces of the human body is considered not significant and hence neglected.

The constitutive relations for stress tensors read as

$\sigma_{f}=-p_{f} I+2 \mu \varepsilon\left(v_{f}\right)$

Where $\mu$ is the dynamic viscosity of the fluid, pf is the Lagrange multiplier corresponding to incompressibility constrain in $(1), \varepsilon\left(\mathrm{v} \_f_{-}\right)$is the strain - rate tensor:

$\varepsilon\left(v_{f}\right)=\frac{1}{2}\left(\nabla v_{f}+\left(\nabla v_{f}\right)^{T}\right)$

The governing equations for the structure are

$\rho_{s}\left(\frac{\partial v_{s}}{\partial t}+\left(\nabla v_{s}\right) v_{s}-g\right)-\nabla \cdot \sigma_{s}=0 \quad \mathrm{IN} \quad \Omega_{t}^{s}$

Where $\mathrm{s}$ denotes structure, $\rho \_s$ is the material density, $g$ represents the external body forces acting on the structure and $\sigma \_s$ is the Cauchy stress tensor.

The structure configuration is described by the displacement us, with velocity field $V_{s}=\frac{\partial u_{s}}{\partial t}$. In a common lagrangian explanation with respect to some fixed position state we have $\rho_{s}\left(\frac{\partial^{2} u_{s}}{\partial t^{2}}-g\right)-\nabla \cdot \Sigma_{s}=0$ In $\Omega_{s}$

Where the tensor $\Sigma_{s}=J \sigma_{s} F^{-T}$ is called the first Piola - Kirchoff tensor (Razzaq et al., 2010) is the momentum equation.

The boundary conditions on the fluid - solid interface is assumed to be $\sigma_{f} n=\sigma_{s} n, v_{f}=v_{s}$ on $\Gamma_{t}^{0}$

In this study, first order high resolution scheme is used initially during steady state analysis to stabilize the flow. The mass flux source coefficient is defined at the interface to achieve convergence and to achieve stability. Sufficiently large pressures were transferred at the interface from fluid to structure. Here mass flux pressure coefficient technique is used with a fixed number of stagger loops and varying values of source coefficients to achieve convergence. Physical time scale is used in the solution at the starting instant during steady state simulation and to achieve convergence with a residual target of 10-5. After steady state simulation, a second order high resolution scheme is utilized to achieve higher order of accuracy for transient simulation using lower courant number automatically from previous steady state results.

\section{Boundary Conditions}

In this study, a three-dimensional carotid artery is studied to elucidate the hemodynamics under different blood pressure conditions. Blood is considered as Newtonian with a dynamic viscosity of 0.004 pas and artery as a linearly elastic model with Elastic modulus of $0.9 \mathrm{E} 6 \mathrm{~Pa}$ and poisons ratio of 0.45 since arterial wall undergoes small deformation approximately $10-15 \%$ of mean diameter [10]. A section from a long artery is considered in this study, therefore in structural model at the nodes at the ends are fixed so that the model is stable during the pulsatile flow. The remaining nodes are free to move due the fluid interaction with the structure. A pulsatile velocity obtained from Doppler ultrasound is applied at the inlet. Resistance boundary condition is applied at the outlet as described by [11]. Figure 1 displays the 3D geometry and the boundary conditions applied.

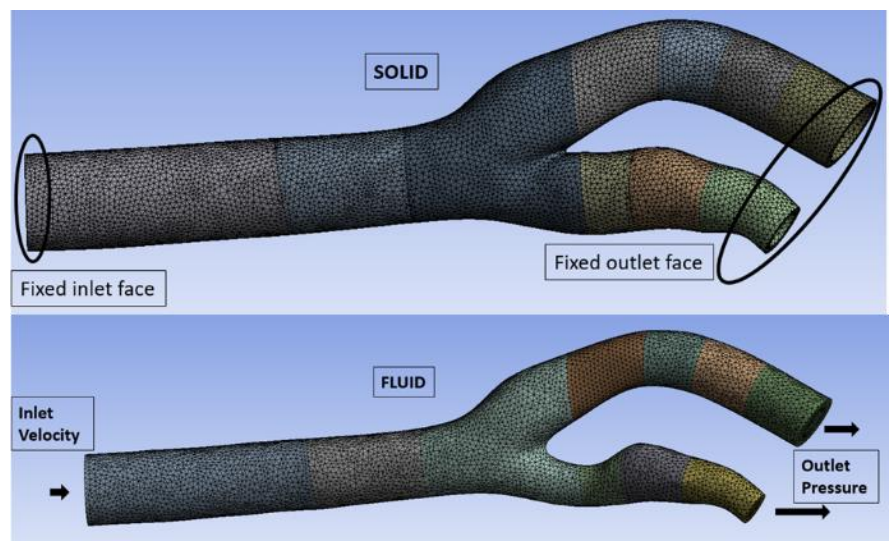

Figure 1: Boundary condition details 3D FSI model

\section{Results and discussion}

Figure 2 displays the analysis of the difference in WSS between non - Newtonian and Newtonian models. Maximum WSS over the entire wall of the artery is noted with a clear observation that the Newtonian model undervalues the WSS 
equated to the Carreau Yasuda model. Vortex core regions were produced during the decelerating phase of the pulsatile flow. Flow diverged while approaching the bifurcation, and then showed helical motion representing vortex formation leading to low shear regions mainly near the carotid sinus and post stenosis in external carotid artery. During this helical flow the stagnation points appeared on wall below the carotid sinus and at the inner wall immediately post stenosis. The region affected due to vortex formation was small during peak systole and higher during late and early diastole.

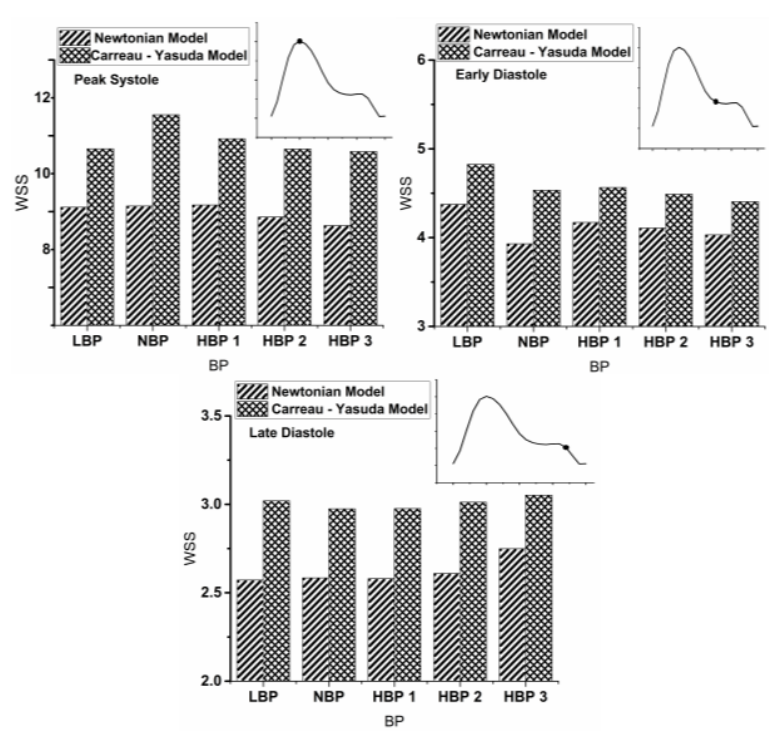

Figure 2: Comparision of WSS between different blood pressures and rheological models.
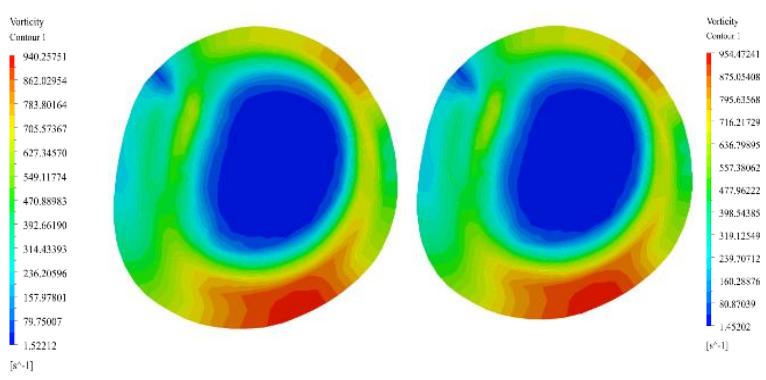

Fig. 3 Vortex core regions for Newtonian and non-Newtonian models

Figure 3 shows the vortex core regions for NBP Newtonian and non-Newtonian viscosity models. If can be enumerated that, although the vortex core regions arer similar qualitatively, there is significant quantitative variance between the two viscosity models.

\section{Conclusions}

The bio-rheological characteristics of blood in arterial flow influences the quantitative behavior of the flow field. The results showed that the Newtonian model is not appropriate for simulating the flow in the carotid artery with stenosis where the rate of shear is less than $100 \mathrm{~s}-1$. The observations in the present study shows the differences in WSS and vorticity and their position was dependent on the flow distribution between the branches and the peripheral resistance due to pressure and also the blood rheology.

\section{References}

[1] Janela, J., Moura, A. and Sequeira, A. (2010b) 'Absorbing boundary conditions for a 3D non-Newtonian fluid-structure interaction model for blood flow in arteries', International Journal of Engineering Science, 48(11), pp. 1332-1349. doi: 10.1016/j.ijengsci.2010.08.004.

[2] Bernard Fox, Keith james, Barbara Morgan, A. S. (1982) 'Distribution of Fatty and Fibrous Plaques in Yung Human Coronary Arteries', Atherosclerosis, 41(2), pp. 337-347.

[3] [3] Giddens, D. P., Zarins, C. K. and Glagov, S. (1993) 'The Role of Fluid Mechanics in the Localization and Detection of Atherosclerosis', Journal of Biomechanical Engineering, 115(4B), p. 588. doi: $10.1115 / 1.2895545$

[4] Markus, H. (2001) 'Severely impaired cerebrovascular reactivity predicts stroke and TIA risk in patients with carotid artery stenosis and occlusion', Brain, 124(3), pp. 457-467. doi: 10.1093/brain/124.3.457.

[5] Eliasziw, M., Kennedy, J., Hill, M.D., Buchan, A.M. and Barnett, H.. (2004) 'Early risk of stroke after a transient ischemic attack in patients with internal carotid artery disease', Canadian Medical Association Journal, 170(7), pp. 1105-1109.

[6] Bakker, P. G. (1991) Bifurcations in Flow Patterns Nonlinear Topics in the Mathematical Sciences. doi: 10.1007/978-94-011-3512-2.

[7] Agarwal, R., Katiyar, V. K. and Pradhan, P. (2008) 'A mathematical modeling of pulsatile flow in carotid artery bifurcation', International Journal of Engineering Science. Elsevier Ltd, 46(11), pp. 1147-1156. doi: 10.1016/j.ijengsci.2008.06.002.

[8] Toloui, M., Firoozabadi, B. and Saidi, M.S., 2012. A numerical study of the effects of blood rheology and vessel deformability on the hemodynamics of carotid bifurcation. Scientia Iranica, 19(1), pp.119126.

[9] Cho, Y. I. and Kensey, K. R. (1991) 'Effects of the non-Newtonian viscosity of blood on flows in a diseased arterial vessel. Part 1: Steady flows.', Biorheology, 28(3-4), pp. 241-62.

[10] [10] Salzar, R. S., Thubrikar, M. J. and Eppink, R. T. (1995) 'Pressureinduced mechanical stress in the carotid artery bifurcation: A possible correlation to atherosclerosis', Journal of Biomechanics, 28(11), pp. 1333-1340. doi: 10.1016/0021-9290(95)00005-3.

[11] Corrada, M.M., Hayden, K.M., Paganini-Hill, A., Bullain, S.S., DeMoss, J., Aguirre, C., Brookmeyer, R. and Kawas, C.H., 2017. Age of onset of hypertension and risk of dementia in the oldest-old: The 90+ Study. Alzheimer's \& Dementia, 13(2), pp.103-110.

[12] Jahangiri, M., Saghafian, M. and Sadeghi, M.R., 2017. Numerical simulation of non-Newtonian models' effect on hemodynamic factors of pulsatile blood flow in elastic stenosed artery. Journal of Mechanical Science and Technology, 31(2), pp.1003-1013.

[13] Lee, B., Kwon, H., Kim, H. and Suh, S., 2018. FSI analysis in stenotic coronary artery model: progress of atherosclerosis and vulnerable hemorheology. Atherosclerosis, 275.

\section{Creative Commons Attribution License 4.0 (Attribution 4.0 International, CC BY 4.0)}

This article is published under the terms of the Creative Commons Attribution License 4.0 https://creativecommons.org/licenses/by/4.0/deed.en US 